\title{
BIBECHANA
}

A Multidisciplinary Journal of Science, Technology and Mathematics

ISSN 2091-0762 (online)

Journal homepage: http://nepjol.info/index.php/BIBECHANA

\section{Acoustoelectric effect in semiconductor superlattice}

\author{
Ujjwal Alok $^{1^{*}}$, Manoj Kumar ${ }^{1}$, B.K.Singh ${ }^{1}$, Praveen Kumar ${ }^{2}$ \\ ${ }^{1}$ Univ. Dept. of Physics, T.M.B. University, Bhagalpur (Bihar) India \\ ${ }^{2}$ Research Scholar, S.K.M. University, Dumka (Jharkhand) India \\ *Corresponding author, E-mail : okujjwal@rediffmail.com
}

Article history: Received 8 July, 2011; Accepted 7 August, 2011

\begin{abstract}
Acoustoelectric effect in semiconductor superlattice $(S L)$ in the region $q />>1$ has been studied. The equation of motion of the lattice has been transformed into a simple form which becomes identical with the dynamic equation for the wave amplitude in the theory of plasma turbulence. A dispersion relation has been derived from the acoustoelectric current $\mathrm{J}$ on the constant electric field $\mathrm{E}$. It is noted that when the electric field is negative the current $\mathrm{J}$ rises, reaches a peak and falls off. On the other hand, when the electric field is positive the current decreases, reaches a peak and then rises. A similar observation has been noted for an acoustoelectric interaction in a multilayered structure resulting from the analysis of the $\mathrm{Si} / \mathrm{SiO}_{2}$ structure.
\end{abstract}

Keywords: acoustoelectric current; acoustomagnetothermal effect; quantum transparency

\section{Introduction}

The Propagation of acoustic wave through a semiconductor has been discussed from many angles. Blotekjaer and Quate [1] have used the coupled mode approach in which one regards the acoustic wave as the lattice mode which are coupled Piezo - electrically to the space charge modes of the electron distribution. In [2] Thakur et al. observed the effect of a dc current on the drift of optically generated carriers in a quantum well. Another interesting mechanism based on the transfer of energy and momentum is the interaction of acoustic phonons with carrier charges in semiconductor materials. This mechanism occurs not only during the scattering of quasimomentum carriers with lattice vibrations but also when acoustic waves propagate through the material. Among the effects observed are the absorption ( amplification ) of acoustic waves [3-5], acoustoelectric effect (AE) [6-10], acoustomagnetoelectric effect (AME) [11-12], acoustothermal effect [13], and acoustomagnetothermal effect [13].

These phenomena have, however, received very little attention in (SL) even though they have immense device applications. Acoustoelectric effect is the transfer of momentum from acoustic waves to the conduction electrons as a result of which may give rise to a current usually called the acoustoelectric current $\mathrm{J}$ or in the case of an open circuit, a constant electric field $\mathrm{E}$. The study of this effect is vital because of the complimentary role it may play in the understanding of the properties of the SL which we believe should find an important place in the acoustoelectric devices. Experimental evidence of the dependence of the acoustoelectric effect on the 
parameters of SL has been reported in [14]. In [15] experimental work on the acoustoelectric interaction of SAW in GaAs- In GaAs superlattice has been reported. A theoretical model for measuring transverse acoustoelectric voltage in multilayered structure resulting from the analysis of the $\mathrm{Si} / \mathrm{SiO}_{2}$ has also been reported in [16].

\section{Distribution Function and Threshold Field}

Assume the sound wave as collimated monochromatic Phonons with all exactly in phase with one another, we write distribution function, as.

$$
f(k)=\delta(k-q)
$$

where ' $k$ ' is the current phonon wave vector, in many semiconductors, such as GaAs the conduction band has a minimum at zone centre and an indirect gap with higher energy at another minimum, at a critical point on the zone boundary. The SL has above other minimum on zone boundary. If the GaAs quantum well width is small enough, the confined state of the zone centre can be pushed higher than the other zone boundary. In this case, electrons will fall from the zone centre into other minimum. The valence band holes will remain confined in this layers, however. This is called a Type -II SL. A structure in which both the electrons and the holes are in the same layer is called a Type $-\mathrm{I} S \mathrm{SL}$. SL have been used to demonstrate the basic effect of Bloch oscillations [17] in which a D C electric field generates.

It is assumed that the sound wave and the applied constant electric field $E$ propagates along the $Z$ - axis of the SL. The problem will be solved in the quasi - classical case, i.e. $2 \Delta>>T^{-1}(\hbar=1)$, eED $<2 \Delta$ ( $D$ is the period of the SL, $2 \Delta$ is the width of the lowest energy miniband and $e$ is the electron charge ). The current density associated with an acoustic wave may be obtained from the expression [18].

$$
\mathrm{J}=\frac{e \Phi}{2 \pi^{2} \omega_{q} v_{\mathrm{g}}} \int U \psi_{i}(P) d^{3} P
$$

where,

$$
U=-\left|G_{p-q, p}\right|^{2}\left[f\left(\epsilon_{p+q}\right)-f\left(\epsilon_{p}\right)\right] \delta\left(\epsilon_{p-q}-\epsilon_{p}+\omega_{q}\right)+\left|G_{p-q, p}\right|^{2}
$$

' $\phi$ 'is the sound flux density, $\omega_{\mathrm{q}}$ and $\mathbf{u}_{\mathrm{s}}$ are the frequency and the group velocity of sound wave with the vector $q$ and $\psi_{i}(P)$ is the solution of the Boltzmann kinetic equation in the absence of a magnetic field. If we introduce a new term $p^{\prime}=p-q$ in the second term of the integrals in Eq. (3) and take $\left|G p^{\prime} p\right|^{2}=\left|G p p^{\prime}\right|^{2}$

We can express Eq. (3) in the form

$$
\mathrm{J}=\frac{-\phi_{\phi}}{2 \pi^{2} \omega_{q} v_{q}} \int|G(p, q)|^{2}\left[f\left(\epsilon_{p+q}\right)-f\left(\epsilon_{p}\right)\right]\left[\Psi_{i}(p+q)-\Psi_{i}(p)\right] \delta\left(\epsilon_{p+q}-\epsilon_{p}-\omega_{q}\right) d^{3} P
$$

where the vector $\psi_{i}(p)$ as indicated in [19] is the mean free path $l_{i}(p)$.

Thus the acoustoelectric current in Eq. (4) in the direction of SL axis becomes

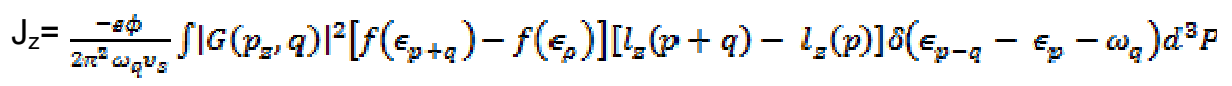

where $f\left(\epsilon_{p}\right)$ is the distribution function, $p$ is the momentum of electrons, $G\left(P_{2}, q\right)$ is the matrix element of the electron-phonon interaction and for $\mathrm{qD}<<1$ it is given as, 


$$
|G(p, q)|^{2}=\frac{|c|^{2} q^{2}}{2 \sigma \omega_{q}}
$$

where $\mathrm{C}$ is the deformation potential constant. $\sigma$ is the density of the SL in the T approximation and further when $\mathrm{T}$ is taken to be constant,

$$
\begin{aligned}
& \mathrm{I}_{\mathrm{z}}=\mathrm{T} \mathrm{S}_{\mathrm{z}} \\
& \mathrm{S}_{\mathrm{z}}=\frac{\partial \varepsilon}{\partial p_{z}}
\end{aligned}
$$

Inserting Eqs. (6) and (7) into Eq. (5) we obtain

$$
J_{z}=-\frac{\nexists \phi|c|^{2} q^{2} \tau}{4 \pi^{2} \sigma v_{s} \omega_{q}^{2}} \int\left[f\left(\epsilon_{p+q}\right)-f\left(\epsilon_{p}\right)\right]\left[s_{z}(p+q)-S_{z}(p)\right] \delta\left(\epsilon_{p+q}-\epsilon_{p}-\omega_{q}\right) d^{3} p
$$

For superlattices the dispersion law is given by

$$
\epsilon_{v}(P)=\frac{P_{z}^{2}}{2 m}+\epsilon_{v}-\Delta_{v} \cos \left(P_{l} D\right)
$$

where $\mathrm{Pt}$ and $\mathrm{Pl}$ are the transverse and longitudinal ( relative to the $\mathrm{SL}$ axis ) components of the quasi momentum respectively, $\Delta \mathrm{U}$ is the half width of the uth allowed miniband

$$
\epsilon_{v}=\frac{\hbar^{2}}{2 m}\left(\frac{\pi^{2}}{d_{0}}\right) v^{2}
$$

are the size- quantized levels in an isolated conduction film, $D=d_{0}+d_{1}\left(d_{0}\right.$ is the width of the rectangular potential wells and $d_{1}$ is the barrier width a non-zero quantum transparency) is the $S L$ period.

The distribution function in the presence of the applied constant field $E$ is obtained by solving the Boltzmann equation in the $\mathrm{T}$ approximation, given by

$$
\begin{array}{ll} 
& \mathrm{f}(\mathrm{P})=\int_{0}^{\alpha} \frac{d l}{\tau} \exp (-t / \tau) f_{0}(P-e E t) \\
\text { Here, } & f_{0}(P)=\frac{\pi d n}{m T_{\mathrm{o}}\left(/_{\mathrm{T}}\right)} \exp \left(-\epsilon_{p} / \mathrm{T}\right)
\end{array}
$$

where $\mathrm{n}$ is the electron density, $\mathrm{T}$ is the temperature in energy units and $\mathrm{I}_{\mathrm{o}}(\mathrm{x})$ is the modified Bessel function.

We assume that electrons are confined to the lowest conduction miniband $(u=1)$ and omit the miniband indices. This is to say that the field does not induce transitions between the filled and empty minibands. The electron velocity is given by

$$
v_{g}(P)=\frac{\partial_{E}(P)}{\partial P_{l}}=\Delta D \sin \left(P_{l} D\right)
$$

We further assume that $\epsilon_{v}=\Delta_{v}$ and write Eq. (10) in the usual form as

$$
\epsilon(P)=\frac{P_{t}^{2}}{2 m}+\Delta\left[1-\cos \left(P_{l} D\right)\right]
$$

Substituting Eqs. (12),(14) and (15) into Eq. (4) and solving for a non-degenerate electron gas. 


$$
\mathrm{J}_{\mathrm{z}}=\frac{|c|^{2} q^{2} n \epsilon \phi\left(b^{2}-1\right) d \tau \theta}{\sigma \omega_{q}^{2} v_{s}} \int_{0}^{\infty} \frac{d t}{\tau} \exp \left(-t^{5} / \tau\right)\{X-X\}
$$

where $\Theta$ is the Heaviside step function.

$$
\begin{aligned}
& \mathrm{b}=\frac{\Delta_{Q}}{2 \Delta \sin \left(\mathrm{Q}^{D} / 2\right)} \\
& X=\operatorname{Sinh}\left[\frac{\Delta}{T} \cos \left(\frac{q D}{2}\right) \cos (e E d t) \sqrt{1-b^{2}}\right] \sinh \left[\frac{\omega_{a}}{2 T} \cos \left(e E d t^{\prime}\right)\right] \text {, and } \\
& X^{\prime}=-2 \frac{\Delta}{\mathrm{T}} \sqrt{1-\mathrm{b}^{2}} \sin \frac{\mathrm{eEdt^{ \prime }}}{2} \sin \left(\frac{\mathrm{qD}}{2}\right) \cos \frac{\mathrm{eXdt^{ \prime }}}{2} \cosh \left[\frac{\Theta_{\mathrm{T}}}{2 \mathrm{~T}} \cos (\mathrm{eEdt})\right] \cdot \cos \left[\frac{\Delta}{\mathrm{T}} \sqrt{1-\mathrm{b}^{2}} \cos \left(\frac{\mathrm{qD}}{2}\right) \cos (\mathrm{eEdt})\right]
\end{aligned}
$$

We shall solve Eq. (16) for two particular case,

(i) in the absence of the applied constant field $(E=0)$, from Eq. (16) we obtain

$\mathrm{J}_{\mathrm{z}}=\frac{|c|^{2} q^{2} n \Theta \phi\left(b^{2}-1\right) d \tau \theta}{4 \sigma \omega_{q}^{2} v_{s}} \sinh \left[\frac{\Delta}{T} \cos \left(\frac{q D}{2}\right) \sqrt{1-b^{2}}\right] \cdot \sinh \left(\frac{\omega \omega_{q}}{2 T}\right)$

If $\omega_{q} \gg 2 \Delta \sin \left(\frac{q D}{2}\right), J_{z}=0$ i.e; there appears a transparency window. This is a consequence of the conservation law.

(ii) In a weak constant electric field, eEd $\tau \ll 1, \omega_{q} \ll T$, from Eq. (16) we obtain

$$
\mathrm{J}_{\mathrm{Z}}=J_{0}\left\{1-\frac{a E d \tau}{\omega_{q}} \sqrt{\left[2 \sin \left({ }^{q D} / 2\right)\right]^{2}-\omega_{q} \operatorname{coth}\left[\frac{\Delta}{T} \cos \left({ }^{q D} / 2\right) \sqrt{1-b^{2}}\right]}\right\}
$$

From Eq. (18) it is observed that at

$$
\mathrm{E}>\mathrm{E}_{0}=\omega_{\mathrm{q}} \frac{\tanh \left[(\Delta / T) \cos (q D / 2) \sqrt{1-b^{2}}\right]}{\operatorname{mD} \sqrt{2 \sin \left(q^{D D} / 2\right)^{2}-\omega_{q}}}
$$

the acoustoelectric current changes sign. The value $E_{0}$ can be interpreted as a threshold field. $E_{0}$ is a function of the SL parameters $D$ and $\Delta$, and temperature $T$, frequency $\omega_{q}$ and the wave number q. For example, at $\Delta / T \ll 1, \Delta=0.1 \mathrm{ev}, \mathrm{D}=5 \times 10^{-7} \mathrm{~cm}, \tau=10^{-12}$, $v_{s}=5 \times 10^{5} \mathrm{cms}^{-1}$ and $\omega_{q}=10^{10} \mathrm{~s}^{-1}$. For these values we obtain the threshold field $\mathrm{E}_{0}=8.65 \mathrm{vcm}^{-1}$ which is small and can be observed.

\section{Results and Discussion}

The general solution of Eq. (16) cannot be obtained analytically. We, therefore, obtained it numerically and the graph of $J_{z}$ on $E$ have been plotted. It is noted that the acoustoelectric current has a peak at some values $E$. These peaks decrease with a corresponding decrease of $\Delta$. More interesting is the nature of the acoustoelectric current. It is observed that when the electric field is negative the current rises and reaches a maximum and then falls of in a manner similar to that 
observed during a negative differential conductivity. On the other hand, when the electric field is positive the current decreases and reaches a minimum then increases. This can be attributed to the Bragg reflection at the band edge. It is further observed that the ratio of the height of the peak corresponding to absorption to that corresponding to amplification differ from one. This value also decreases with a decrease in $\Delta$. The threshold field $E_{0}$ also increases with the decrease of $\Delta$. It is noteworthy to show that a similar relation was obtained for a transverse acoustoelectric voltage experiment on $\mathrm{Si} / \mathrm{SiO}_{2}$ and this result agrees quite well with our result [15]

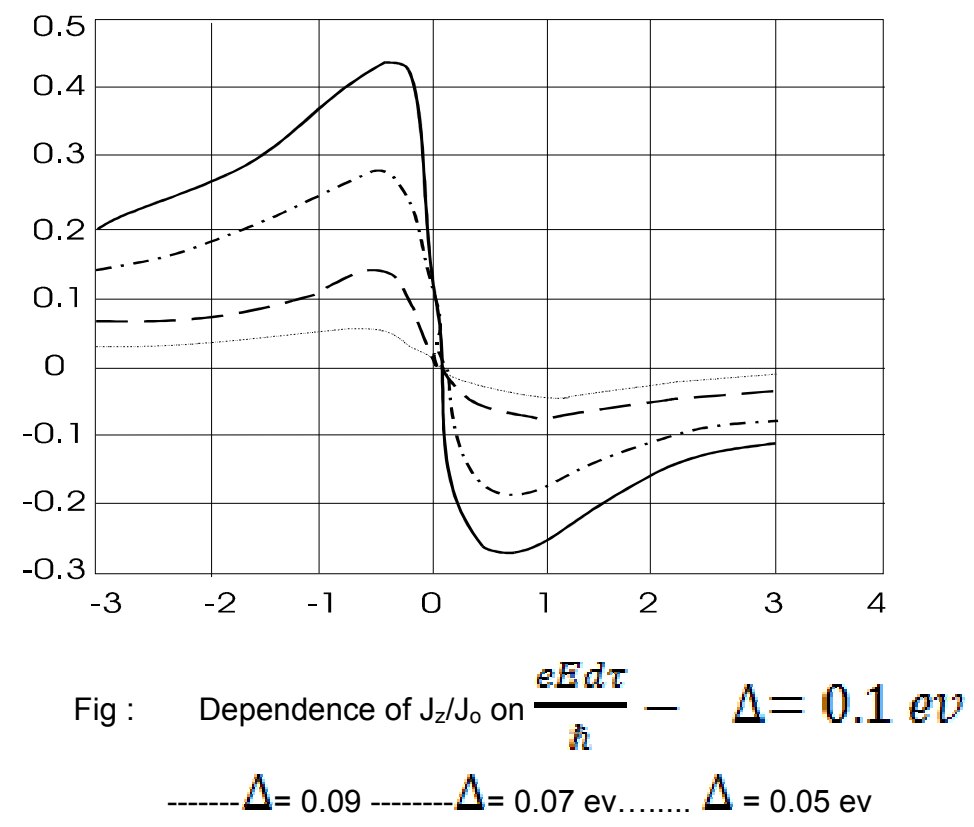

\section{References}

[1] K. Blotekjaer and C.F.Quate, Proc. IEEE 52 (1964) 360

[2] R. Y. Thakur, B. K. Singh. and Anjula Singh, Semicond.Sci. Technol. Uk 5 (1990) 405

[3] K.B.Tolpygo and Z.I.Uritskii, Zh. Eksp. Teor. Fiz 30 (1956) 929

[4] G. Weinreich, Phys. Rev. 104 (1956) 321

[5] A.R.Hatson, J.H McFee and D.L.White, Phys. Rev. Lett. 7 (1961) 237

[6] R.H.Parmenter, Phys. Rev. B 89 (1953) 990

[7] M. Rotter, A.V.Kalameitsev, A.O.Govorov, W. Ruile and A. Wixforth, Phys. Rev. Lett. 82 (1999) 2171

[8] J. M. Shilton, D.R.Mace, V.I. Talyanskii, Yu. Galperin, M.Y.Simmons, M.Pepper and D.A.Ritchie, J.Condens. Matter 8 (1996) L 337

[9] V.L.Gurevich and V.I. Kozub, Phys. Rev. B 58 (1998) 13088

[10] V.V.Afonin, Yu. M. Gal'perin, Semiconductor 27 (1993) 61

[11] M.I.Kaganov, Sh. T. Mevlyut and I.M. Suslev, Sov. Phys JETP 51 (1980) 189

[12] A.D. Margulis and V.A.Margulis J.Condens. Matter 6 (1994)6139

[13] Yu. V. Gulyaev and E.M.Epshlein Sov. Phys. Solid State 9 (1967) 674

[14] V.A Vyun, Yu.O. Kanter, S.M.Kikkarin, V.V.Pnev, A.A.Fedorov, and Yakovkin 1988, Academy of Science USSR $11^{\text {th }}$ All union conference on Physics of Semiconductors Kishinev, 1988) Vol 3 (Kishinev Academy) p118 
Ujjal Alok et al. / BIBECHANA 8 (2012) 67- 72: BMHSS, p.72

[15] V.A Vyun, Yu.O.Kanter, S.M. Kikkarin, V.V.Pnev, A. A.Fedorov, and I.B.Yakovkin, Solid State Communications 78 (1991) 823

[16] F. Palma, J.Appl. Phys. 66 (1989) 292

[17] C. Waschke, Physical Review Letters 70, (1993) 3319

[18] M.I. Kaganov. Sh.T. Mevlyut, and I.M.Suslev, Zh. Eksp. Teor. Fiz 78 (1980) 376

[19] S.V. Krychkov and N.P. Mikheev, Fiz. Tekh. Poluprov. 16 (1982) 169 